\title{
Early experience of nintedanib in COVID-19 ARDS-related pulmonary fibrosis: a case series
}

\begin{abstract}
The current COVID-19 pandemic has spread like wildfire worldwide and has affected millions of people. The novel corona virus mainly affects the lungs leading to life threatening disease like acute respiratory distress syndrome (ARDS). The aftermath of the disease in form of pulmonary fibrosis is upcoming cause of further increase in morbidity and mortality. Nintedanib is an oral antifibrotics with proven role in idiopathic pulmonary fibrosis, however its use in COVID-19 related pulmonary fibrosis has not been studied. We report our early experience of use of nintedanib in COVID-19 related pulmonary fibrosis.
\end{abstract}

Key words: SARS-CoV-2, ARDS, pulmonary fibrosis, antifibrotics, nintedanib

Adv Respir Med. 2021; 89: 589-596

\section{Introduction}

The current SARS-CoV-2 pandemic has affected millions of people worldwide. It mainly affects the lung progressing to respiratory failure. Post COVID-19 ARDS related pulmonary fibrosis is an important entity which is posing a management dilemma to the clinicians. The pulmonary fibrosis begins early in the course of ARDS and is more common in patients with longer duration of ICU stay [1,2]. The risk factors constitutes advanced age, male gender, underlying co-morbidities like diabetes and patients with severe COVID-19 disease [3-5]. The pathophysiology of pulmonary fibrosis in COVID-19 patients includes dysregulated immune mechanisms which causes generalized epithelial and endothelial injury and finally an aberrant healing process leading to pulmonary fibrosis $[1,6,7]$. Nintedanib is currently used widely in management of idiopathic pulmonary fibrosis and prevents the decline in lung function [8-10]. Here we present our early experience of use of nintedanib in COVID-19 related pulmonary fibrosis.

\section{Case series}

This is a case series of four patients carried admitted at our tertiary care hospital in western Maharashtra from April 2020 to October 2020. All four patients were confirmed COVID-19 cases by nasopharyngeal swab RT-PCR test.

\section{Case 1}

A 62-years-old female with no known co-morbidities, reported to emergency with history of fever and cough of five days and insidious onset breathlessness of one day duration. She was diagnosed as COVID-19 infection on her nasopharyngeal RT PCR test. She reported to the hospital on seventh day of symptoms and on arrival, she was tachypnoeic and her saturation was $78 \%$ on room air which improved to $90 \%$ with oxygen through non- rebreather mask (NRBM) at 15 litres/min. On evaluation, she had mild anaemia (Hb-12 gm/dL) with lymphopenia and high neutrophil/lymphocyte ratio (N/L-8). Her liver enzymes and renal function tests were within normal limits. Her chest radiograph showed

Address for correspondence: Robin Choudhary, Army Institute of Cardiothoracic Sciences, Department of pulmonary, Critical Care and Sleep medicine, 411040 Pune, India, e-mail: robinch19@gmail.com

DOI: 10.5603/ARM.a2021.0113 | Received: 07.05.2021 | Copyright (C) 2021 PTChP | ISSN 2451-4934 | e-ISSN 2543-6031

This article is available in open access under Creative Common Attribution-Non-Commercial-No Derivatives 4.0 International (CC BY-NC-ND 4.0) license, allowing to download articles and share them with others as long as they credit the authors and the publisher, but without permission to change them in any way or use them commercially. 

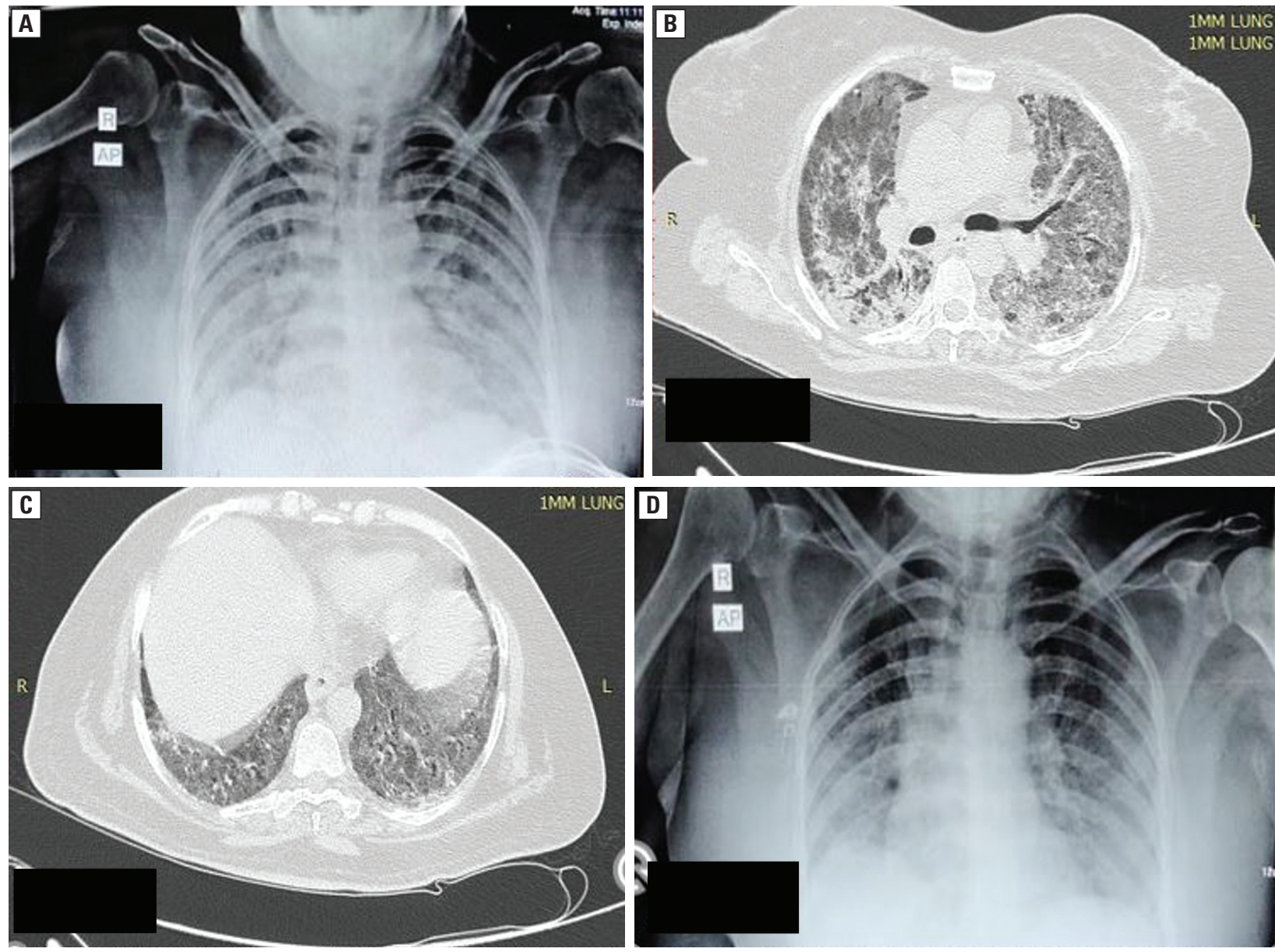

Figure 1A. Chest skiagram showing bilateral airspace opacities in mid and lower zones; B, C. High resolution computed tomogram of chest showing pulmonary fibrosis in bilateral lower lobes; D. Chest skiagram showing improvement after 3 weeks of antifibrotics therapy

bibasal peripheral airspace opacities (Figure 1A). She was managed with injectable antibiotics, steroids (Inj dexamethasone $6 \mathrm{mg}$ once daily for 10 days), therapeutic dose of low molecular weight heparin (LMWH) and oxygen therapy. However, she had progression of disease to acute respiratory distress syndrome (ARDS) and was not maintaining saturation on high flow oxygen, therefore was commenced on non-invasive ventilation (NIV) (settings: pressure support $-4 \mathrm{~cm}$ $\mathrm{H}_{2} 0$, PEEP - 10-12 $\mathrm{cm} \mathrm{H}_{2} 0, \mathrm{FiO}_{2}$ - on titration with target saturation $88-92 \%$ ). Her arterial blood gas showed hypoxemia with low partial pressure of oxygen $\left(\mathrm{pO}_{2}\right)$ to fraction of inspired oxygen $\left(\mathrm{FiO}_{2}\right)-\left(\mathrm{pO}_{2} / \mathrm{FiO}_{2}\right)$ less than 100. She also showed features of hyperinflammation in form of raised LDH, ferritin, CRP and IL-6 (Table 1) for which she was administered 2 doses of tocilizumab ( $8 \mathrm{~mL} / \mathrm{kg}), 24$ hours apart. She was also given two doses of COVID convalescent plasma (CCP) (200 mg/dose), 24 hours apart. The patient showed significant response and was gradually weaned off NIV but she continued to have high oxygen requirement. Her chest roentgenogram showed dense fibrotic opacities in bilateral lower zones. She underwent a high resolution computed tomography (HRCT) of chest which showed severe involvement of bilateral lung with fibrosis in bilateral lower zones which was more on the right side (Figure 1B, C). She was diagnosed as post COVID-19 ARDS pulmonary fibrosis and was commenced on oral tablet nintedanib $(150 \mathrm{mg}$ twice daily). She showed significant response and was gradually weaned off high flow nasal oxygen after 3 weeks and was discharged on minimal domiciliary oxygen supplementation. The follow-up chest roentgenogram showed significant clearing of fibrotic opacities (Figure 1D).

\section{Case 2}

A 36-years old male with no known co-morbidities, initially reported with history of fever and cough of three days duration and history of breathlessness of one day duration. On arrival, he was tachypnoeic (respiratory rate $-32 / \mathrm{min}$ ) and hypoxic at room air $(82 \%$ at room air and 


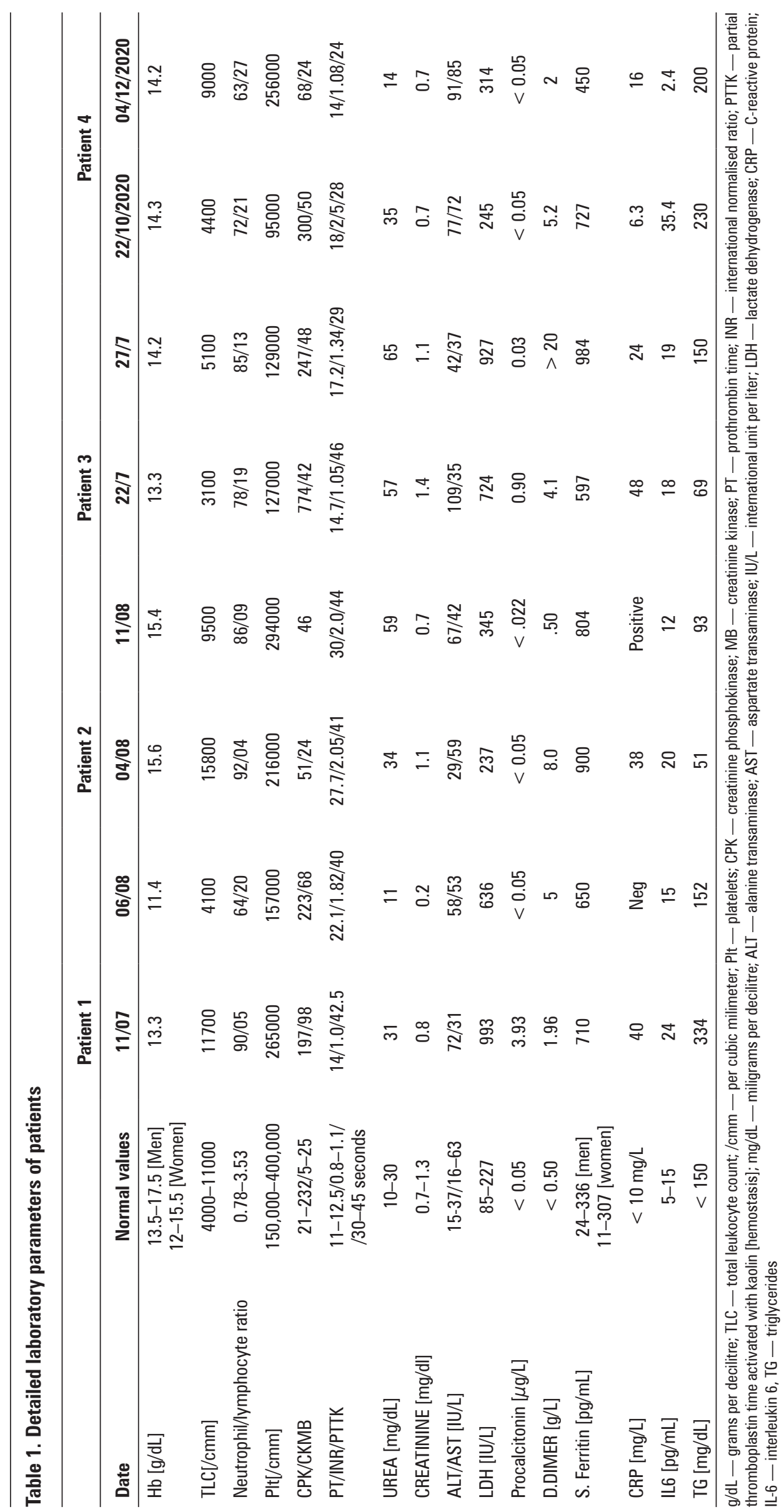



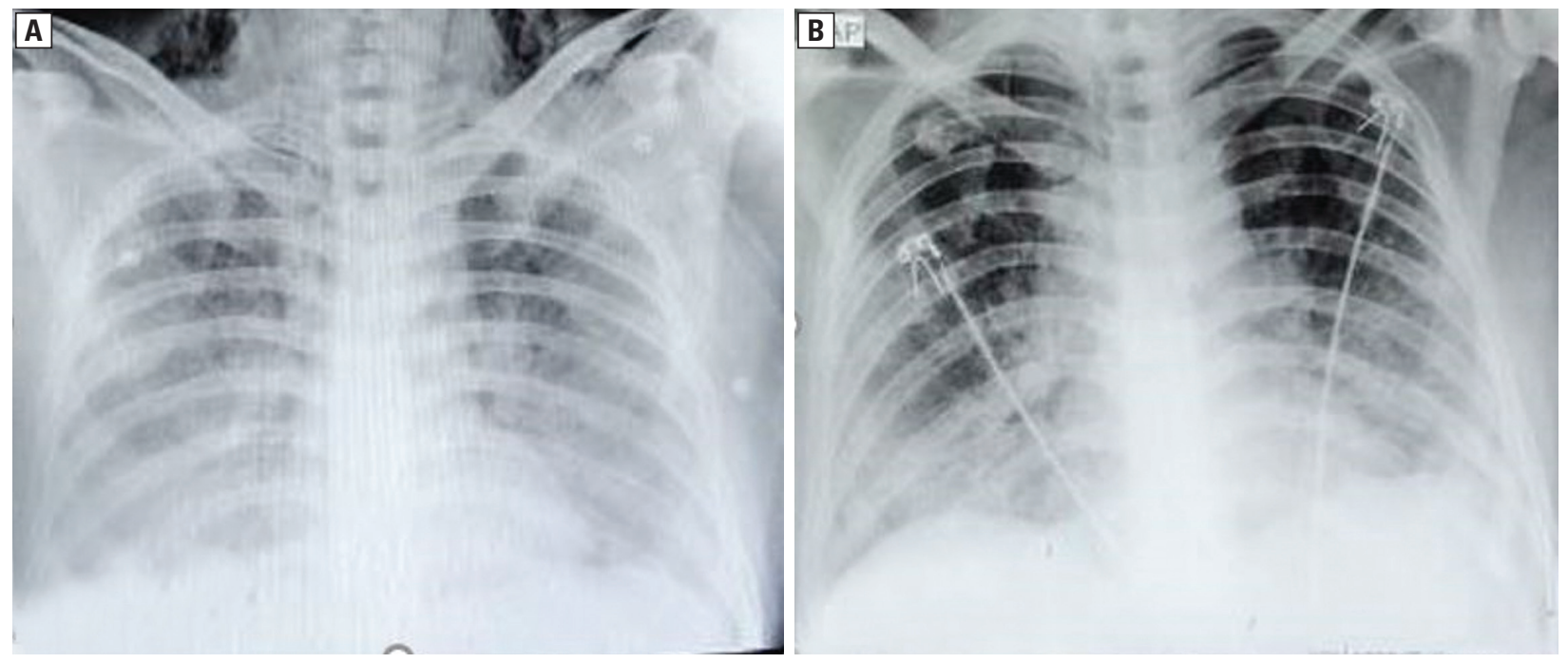

Figure 2A. Chest skiagram showing bilateral airspace opacities involving all the lobes; B. Chest skiagram showing radiological improvement after 3 weeks of nintedanib

Table 2. Arterial blood gas details of COVID-19 patients pre and post (4 weeks) nintedanib

\begin{tabular}{lcccccccc}
\hline & \multicolumn{2}{c}{ Patient 1 } & \multicolumn{2}{c}{ Patient 2 } & \multicolumn{2}{c}{ Patient 3 } & \multicolumn{2}{c}{ Patient 4 } \\
\hline & Day 1 & Day 10 & Day 1 & Day 8 & Day 1 & Day 6 & 22/10/2020 & 04/12/2020 \\
\hline $\mathrm{pH}(7.35-7.45)$ & 7.3 & 7.38 & 7.42 & 7.45 & 7.36 & 7.42 & 7.39 & 7.45 \\
$\mathrm{pCO}_{2}(\mathrm{mmHg})(35-45)$ & 38 & 38 & 32 & 38 & 40 & 38 & 36 & 38 \\
$\mathrm{pO}_{2} \mathrm{mmHg}(80-100)$ & 40 & 55 & 50 & 58 & 40 & 56 & 52 & 58 \\
$\mathrm{HCO}_{3}(\mathrm{mEq} / \mathrm{L})(22-26)$ & 18 & 23 & 23 & 25 & 20 & 24 & 22 & 25 \\
\hline
\end{tabular}

94\% with oxygen through NRBM). On evaluation, he had lymphopenia (total leukocyte count-3100/Cumm)withhighneutrophil/leukocyteratio (> 3.8). He also had raised markers of hyperinflammation (LDH, ferritin, D-dimer and interleukin 6 levels) (Table 1). His chest roentgenogram showed severe COVID pneumonia with bilateral air space opacities and consolidation in mid and lower zones (Figure 2A). His arterial blood gas showed features of type-1 respiratory failure with ARDS $\left(\mathrm{pH}-7.42, \mathrm{pO}_{2}-45, \mathrm{pCO}_{2}-28\right.$, $\left.\mathrm{HCO}_{3}-24\right)$. He was managed as a case of severe COVID-19 pneumonia with injectable antibiotics, parenteral steroids (Inj dexamethasone $6 \mathrm{mg}$ once daily for 10 days), therapeutic dose of LMWH and oxygen therapy through NRBM@15 litre/min. However, his respiratory parameters worsened requiring non-invasive ventilation (settings: Pressure support - 2-4 cm $\mathrm{H}_{2} 0$, PEEP - 10-12 cm $\mathrm{H}_{2} \mathrm{O}, \mathrm{FiO}_{2}$ - on titration with target saturation 88-92\%). He showed favourable response but continued to have high oxygen demand. He was diagnosed as having COVID-19 related pulmonary fibrosis and started on nintedanib (150 mg twice daily) and he showed significant response with improvement in oxygenation after four weeks (Figure 2B, Table 2).

\section{Case 3}

72-year-old male, non-smoker, known case of type-II diabetes mellitus and primary hypertension reported with history of fever, cough and generalised malaise of three days and history of increased breathlessness of one day duration. On arrival he was tachypnoeic (respiratory rate - 35/min) and hypoxic at room air (saturation at room air - 80\%). On evaluation, he had normal complete blood count, liver and kidney function tests but had raised serum markers of hyperinflammation. He was managed with parenteral steroids (Inj dexamethasone $6 \mathrm{mg}$ once daily for 10 days), IV antibiotics, subcutaneous LMWH and oxygen therapy through NRBM@15 litres/min. Hid ABG showed features of type-1 respiratory failure and ARDS $\left(\mathrm{pO}_{2} / \mathrm{FiO}_{2}<100\right)$. He was also given two doses of injection tocilizumab for cyto- 

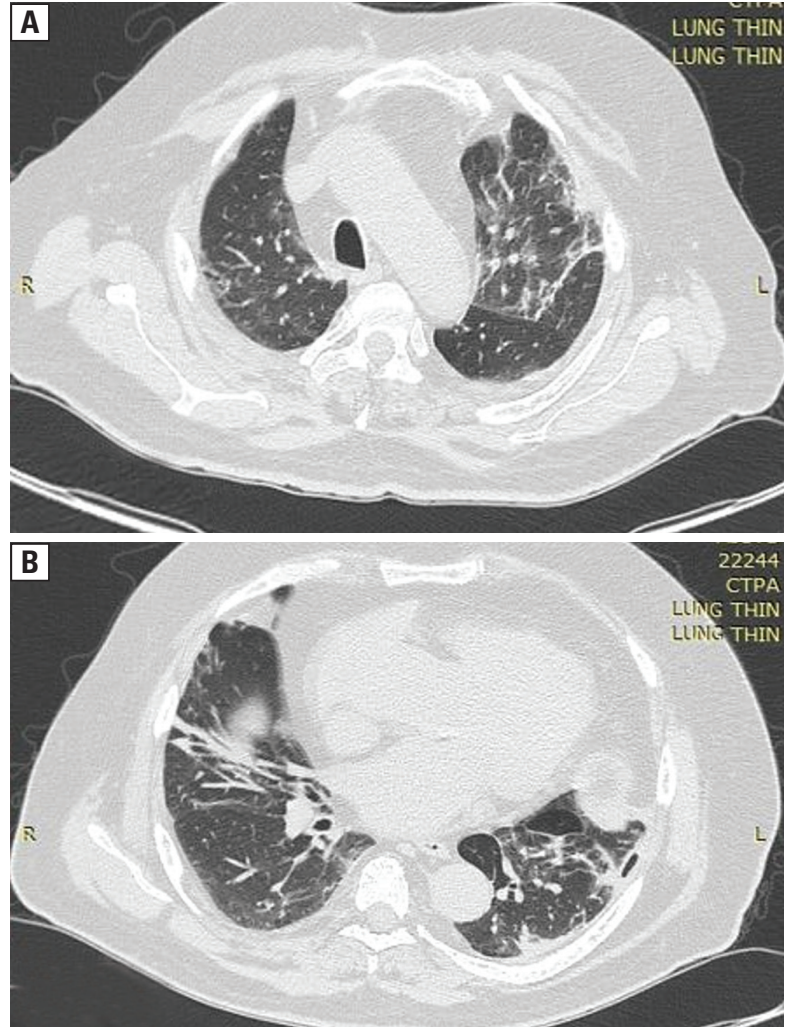

Figure 3A, B. High resolution computed tomography of chest showing fibrotic opacities in bilateral upper lobes (left $>$ right)

kine storm syndrome. However, even after three weeks of intensive management, he continued to have high oxygen requirement. His HRCT of chest showed moderate involvement of bilateral lung with post COVID-19 changes in form of dense fibrosis in bilateral upper lobe (Figure 3A, B). He was started on oral nintedanib and he showed significant improvement and was weaned off oxygen after four weeks.

\section{Case 4}

52-year-old male, known case of type-II diabetes mellitus on oral hypoglycaemic agents, presented with history of fever with chills and dry cough of four days duration. he reported to emergency department on sixth day of symptoms, with history of increased breathlessness. On arrival he was hypoxic (saturation at room air - 82\% and 92\% with oxygen through NRBM@ 15 litres/min). On examination, he was febrile and maintaining saturation at room air (94\%). On evaluation, he had normal complete blood count, liver and renal parameters. However, he had raised parameters of hyperinflammation (Table 1). His arterial blood gas showed features of type- 1 respiratory failure with ARDS $\left(\mathrm{pH}-7.39, \mathrm{pO}_{2}-36, \mathrm{pCO}_{2}-52, \mathrm{HCO}_{3}-22\right)$.
His chest skiagram showed bilateral diffuse peripheral airspace opacities and HRCT of chest showed peripheral ground glass opacities with an apico-basal gradient. He was managed with inj dexamethasone (6 mg once daily for 14 days), antibiotics, intermediate dose of LMWH (Inj LMWH $40 \mathrm{mg}$ Subcutaneous route twice daily) and NIV (settings: pressure support $-2-4 \mathrm{~cm} \mathrm{H}_{2} 0$, PEEP - 10-12 $\mathrm{cm} \mathrm{H}_{2} 0, \mathrm{FiO}_{2}$ - on titration with target saturation $88-92 \%$ ). He showed good response initially but later there were features of disease progression in form of dyspnoea and increased oxygen requirement for which he was started on NIV (settings: pressure support $-2-4 \mathrm{~cm} \mathrm{H}_{2} 0$, PEEP - 10-12 $\mathrm{cm} \mathrm{H}_{2} \mathrm{O}, \mathrm{FiO}_{2}-100 \%$ initially later on titration with target saturation $88-92 \%$ ). He was managed with increased dose of steroids (Inj dexamethasone $6 \mathrm{mg}$ twice daily and therapeutic dose of LMWH. He showed improvement and his steroids were tapered off but he continued to have oxygen requirements. Computed tomography pulmonary angiography (CTPA) was done which did not reveal any evidence of pulmonary embolism but showed features of severe involvement of bilateral lung and interlobular and interseptal thickening with traction bronchiectasis (Figure 4A, B). He was started on antifibrotics (nintedanib tablet $150 \mathrm{mg}$ twice daily). He has completed four weeks of antifibrotics and has shown significant response and is presently maintaining saturation at room air (Figure 4C, D).

\section{DISCUSSION}

The current SARS-CoV-2 pandemic has spread like wildfire and has affected millions of people around the world. COVID-19 primarily affects lungs and has a varied presentation including organising pneumonia to severe lung injury inform of acute respiratory distress syndrome [China, and has subsequently spread worldwide. Risk factors for the clinical outcomes of COVID-19 pneumonia have not yet been well delineated. Objective To describe the clinical characteristics and outcomes in patients with COVID-19 pneumonia who developed acute respiratory distress syndrome (ARDS]. Post COVID-19 pulmonary fibrosis is an upcoming important entity causing increased mortality and morbidity in these patients [2-4].

The risk factors for developing post COVID-19 fibrosis includes old age, severe illness, prolonged ICU stay, requiring mechanical ventilation and those who had significant history of smoking and alcohol consumption [5]. In our 

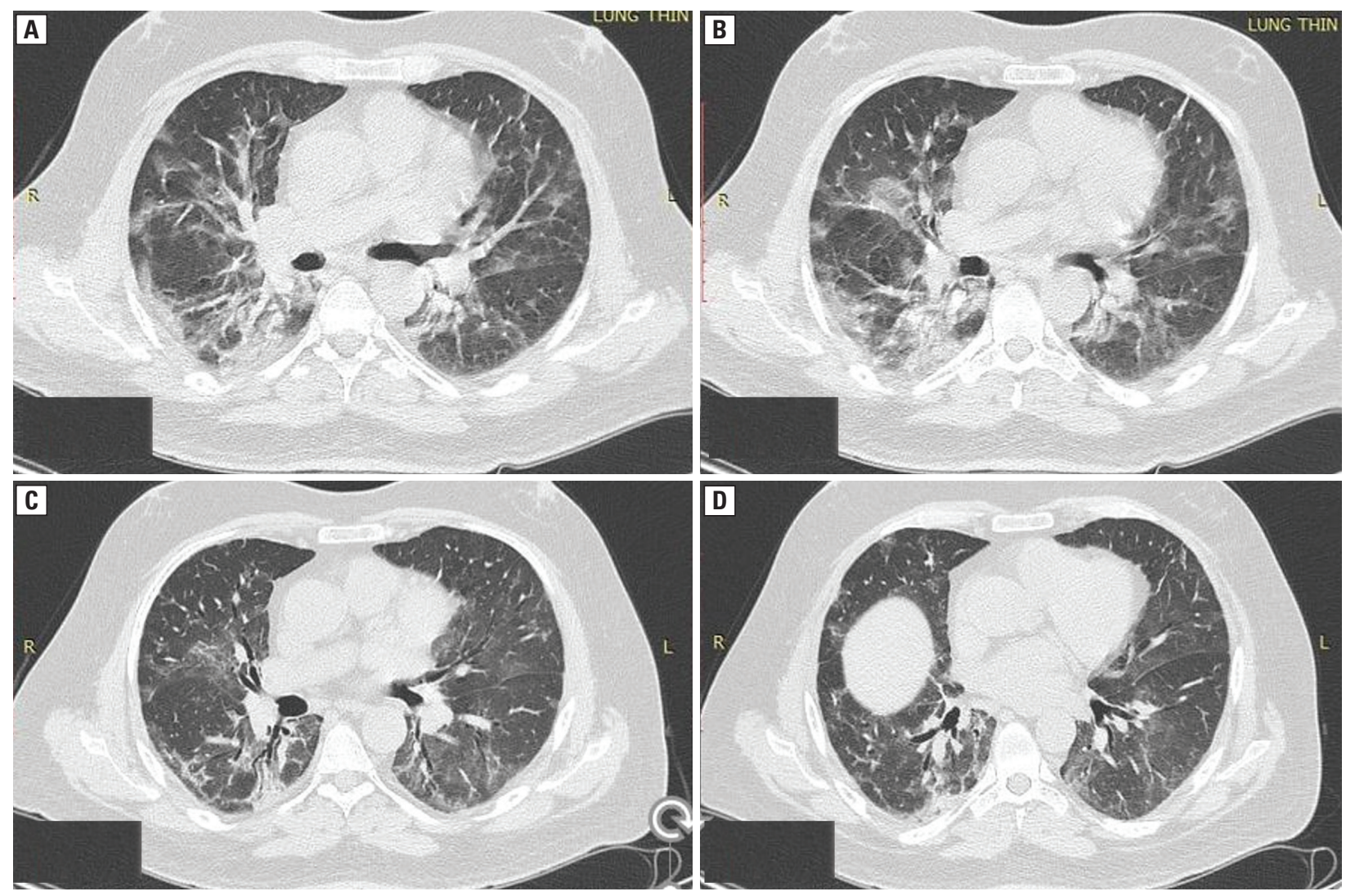

Figure 4A, B. High resolution computed tomography of chest showing bilateral consolidation with traction bronchiectasis; C, D. High resolution computed tomography of chest showing clearing of fibrotic opacities after 4 weeks of nintedanib

study, two of the patients had diabetes while the others had no comorbidities and the development of pulmonary fibrosis was the cause of prolonged hospital stay and difficult weaning.

The pathophysiology of pulmonary fibrosis includes variable mechanism like direct endothelial injury, alveolar epithelial damage, cytokine mediated damage, dysregulated release of matrix metalloproteinases and uncontrolled fibroproliferation. The severe form of ARDS is marked by higher release of cytokines and inflammatory markers which predisposes to higher incidence of pulmonary fibrosis. These factors stimulate the hyperproliferation of type-II vesicular endothelial cells which causes release of fibroblasts and their maturation into myofibroblasts, leading to excessive extracellular matrix accumulation and ultimately fibrosis and alveolar dysfunction [6-8]. This correlates with the duration of disease and is generally seen more in patients with a disease duration more than 3 weeks [7].

All our patients presented with moderate to severe COVID pneumonia and were optimally managed with steroids, LMWH and oxygen therapy. The patients during the course of admission showed progression to ARDS and three patients required non-invasive ventilator support. They showed good response but even after maximal therapy continued to have high oxygen requirement and were difficult to wean off the oxygen support leading to prolonged hospital stay. HRCT chest can confirm the presence of fibrosis in form of reticulations, interlobular and interseptal thickening, traction bronchiectasis, honeycombing and volume loss on the involved side [7, 8]. On functional evaluation, they usually have restriction on spirometry with decreased diffusion capacity of lungs for carbon monoxide (DLCO) and reduced total lung capacity (TLC) [7, 9]. These patients can also have reduced exercise tolerance which can be demonstrated on a 6 minute walk test (6MWT).

The patients of moderate to severe COVID-19 pneumonia with prolonged intensive care unit stay should be suspected of having pulmonary fibrosis. However other causes of increased oxygen requirements like pulmonary thromboembolism should be ruled out by doing a base line CTPA and these patients should preferably should have a negative or reduced C-reactive protein levels and other hyperinflammatory markers which otherwise will benefit more in 
combination with other modalities like prolonging the duration of steroids [2]. All our patients had shown good response with steroids and CTPA done in the patient did not show any features of pulmonary thromboembolism but they continued to have high oxygen requirement which indicated clinical diagnosis of post COVID fibrosis which was confirmed on radiology.

There is great uncertainty in management of COVID-19 pulmonary fibrosis mainly due to non-availability of treatment modalities [2, 6]. Antifibrotics agents like pirfenidone and nintedanib have an established role in fibrotic diseases like idiopathic pulmonary fibrosis (IPF) and they can be used in post COVID-19 fibrosis by that analogy [10, 11]. They have been proven to reduce the rate of deterioration of forced vital capacity (FVC) in patients with IPF $[12,13]$. These drugs do not ameliorate the SARS-CoV-2 immune dysregulation neither address the catastrophic pro-thrombotic state of this infection but can address the development of pulmonary fibrosis post COVID-19 pneumonia. Nintedanib is a tyrosine kinase inhibitor which has been extensively evaluated for its antifibrotics role in IPF [9, 11, 13]. It has displayed anti-angiogenesis properties through blockade of the vascular endothelial growth factor (VEGF) pathway. Nintedanib has shown significant antifibrotic role and has potential anti IL- 1 and IL-6 activity which are one of the main culprits in cytokine storm and post COVID fibrosis [8-10]. The main side effect of nintedanib includes diarrhoea, nausea, reduced appetite and deranged liver function tests [9, 14]. We chose nintedanib for these patients as it has significant immunomodulatory, and anti- inflammatory and anti-thrombotic action in addition to it triple kinase inhibitor activity.

All our patients were started on oral tablet nintedanib and was well tolerated. The decision to start anti-fibrotic was taken as patients, after showing an initial response, failed to show significant clinical improvement, with negative inflammatory markers and continued to be oxygen dependant. HRCT showed evidence of pulmonary fibrosis and they were initiated on anti-fibrotics. All our patients had different extent of pulmonary involvement however they all had severe COVID pneumonia and post COVID pulmonary fibrosis which responded to nintedanib. The exclusion criteria for starting nintedanib includes deranged liver function tests, recent myocardial infarction or haemorrhagic stroke, active haemoptysis or gastrointestinal bleed and deranged coagulation profile. At present there is no biomarker to prompt the administration of anti-fibrotic and the presence of fibrosis on CT scan has been agreed as the best indicator to start therapy [15]. This is our initial experience of using nintedanib in post COVID-19 fibrotic lung disease; however it needs bigger randomized controlled trials to assess the safety and efficacy of this drug.

\section{Conflict of interest}

\section{None.}

\section{References:}

1. Wu C, Chen X, Cai Y, et al. Risk factors associated with acute respiratory distress syndrome and death in patients with coronavirus disease 2019 pneumonia in Wuhan, China. JAMA Intern Med. 2020; 180(7): 934-943, doi: 10.1001/jamainternmed.2020.0994, indexed in Pubmed: 32167524.

2. George PM, Wells AU, Jenkins RG. Pulmonary fibrosis and COVID-19: the potential role for antifibrotic therapy. Lancet Respir Med. 2020; 8(8): 807-815, doi: 10.1016/S22132600(20)30225-3, indexed in Pubmed: 32422178.

3. Lechowicz K, Drożdżal S, Machaj F, et al. COVID-19: the potential treatment of pulmonary fibrosis associated with SARS-CoV-2 infection. J Clin Med. 2020; 9(6), doi: 10.3390/ icm9061917, indexed in Pubmed: 32575380.

4. Spagnolo P, Balestro E, Aliberti S, et al. Pulmonary fibrosis secondary to COVID-19: a call to arms? The Lancet Respiratory Medicine. 2020; 8(8): 750-752, doi: 10.1016/s22132600(20)30222-8.

5. Ojo AS, Balogun SA, Williams OT, et al. Pulmonary fibrosis in COVID-19 survivors: predictive factors and risk reduction strategies. Pulm Med. 2020; 2020: 6175964, doi: 10.1155/2020/6175964, indexed in Pubmed: $\underline{32850151 .}$.

6. Seifirad S. Pirfenidone: A novel hypothetical treatment for COVID-19. Med Hypotheses. 2020; 144: 110005, doi: 10.1016/j. mehy.2020.110005, indexed in Pubmed: 32575019.

7. Vasarmidi E, Tsitoura E, Spandidos DA, et al. Pulmonary fibrosis in the aftermath of the COVID-19 era (Review). Exp Ther Med. 2020; 20(3): 2557-2560, doi: 10.3892/etm.2020.8980, indexed in Pubmed: 32765748.

8. Wang J, Wang BJ, Yang JC, et al. [Research advances in the mechanism of pulmonary fibrosis induced by coronavirus disease 2019 and the corresponding therapeutic measures]. Zhonghua Shao Shang Za Zhi. 2020; 36(8): 691-697, doi: 10.3760/ cma.j.cn501120-20200307-00132, indexed in Pubmed: 32174095.

9. Mo X, Jian W, Su Z, et al. Abnormal pulmonary function in COVID-19 patients at time of hospital discharge. Eur Respir J. 2020; 55(6), doi: 10.1183/13993003.01217-2020, indexed in Pubmed: 32381497.

10. Rivera-Ortega P, Hayton C, Blaikley J, et al. Nintedanib in the management of idiopathic pulmonary fibrosis: clinical trial evidence and real-world experience. Ther Adv Respir Dis. 2018; 12: 1753466618800618, doi: 10.1177/1753466618800618, indexed in Pubmed: $\underline{30249169}$.

11. Kolb M, Richeldi L, Behr J, et al. Nintedanib in patients with idiopathic pulmonary fibrosis and preserved lung volume. Thorax. 2017; 72(4): 340-346, doi: 10.1136/thoraxjnl-2016-208710, indexed in Pubmed: 27672117.

12. King TE, Bradford WZ, Castro-Bernardini S, et al. ASCEND Study Group. A phase 3 trial of pirfenidone in patients with idiopathic pulmonary fibrosis. N Engl J Med. 2014; 370(22): 2083-2092, doi: 10.1056/NEJMoa1402582, indexed in Pubmed: 24836312.

13. Richeldi L, Kolb M, Jouneau S, et al. Efficacy and safety of nintedanib in patients with advanced idiopathic pulmonary fibrosis. BMC Pulm Med. 2020; 20(1): 3, doi: 10.1186/s12890019-1030-4, indexed in Pubmed: 31914963.

14. Bendstrup E, Wuyts W, Alfaro T, et al. Nintedanib in idiopathic pulmonary fibrosis: practical management recommendations 
for potential adverse events. Respiration. 2019; 97(2): 173-184, doi: 10.1159/000495046, indexed in Pubmed: 30544129.

15. Udwadia ZF, Koul PA, Richeldi L. Post-COVID lung fibrosis: The tsunami that will follow the earthquake. Lung India. 2021; 38(Supplement): S41-S47, doi: 10.4103/lungindia.lungindia 818 20, indexed in Pubmed: 33686978. 